\title{
A Review of Nanotechnology Applications
}

\author{
Nick Ledbetter and Tarun Goswami \\ Mechanical Engineering Department \\ Arkansas Tech University \\ Russellville, AR 72801, USA
}

\begin{abstract}
A review of current technology research efforts has been conducted in this paper. In nanoscience, objects are measured in nanometers, 1 billionth of a meter. For comparison, a human hair is approximately 100,000 nanometers thick and the smallest feature on current computer chips measures about 200 nanometers. Since nanotechnology has a wide range of applications from computer chip manufacturing, new materials, renewable energy, interconnection technology to mount the nano-chips, and in medical science, this review presents a broad summary of development efforts in the government and private sector laboratories. Therefore this project on the one hand prepares a review of literature on nanotechnology, and on the other hand attempts to develop a conceptual model of force-displacement analysis in a layer of atoms with a known pattern and atomic bonding force between them when an external force is exerted. The model will make assumptions of the bonding forces between them and predict a path in which atoms will be displaced. Such a model will integrate and develop more knowledge on the failure and fracture of nanosystems.
\end{abstract}

\section{INTRODUCTION}

Nanotechnology is the study of materials and structures and manufacturing of such components at nanoscale. In order to understand this subject, four common areas were selected and those areas will be reviewed in this paper. The four areas are: 1. concept modeling of nano-scale atoms, 2. fabrication aspects of overlaying layered structures, 3. imaging of nano-scale structures and 4. failure analysis of nano-structures. Therefore, topics that are of interest to the Nanotechnology group at Arkansas Tech are X-ray crystallography, atomic physics, and laying of nano-scale layered structures by different physical vapor deposition methods and/or other methods.

Nanotechnology is important in such areas as material science, mechanical engineering, aerospace, medicine, and computer technology. Even though nano-lithography has been in practice for some time, yet precise manufacturing of computer chips requires knowledge of nano-scale manufacturing. This paper will 
focus on an overview of current technology in the four areas identified earlier, conceptual modeling, imaging, material properties, and possible applications, which include medicine, aerospace, and computers.

\section{CONCEPTUAL MODELING}

The continuation of present trends in computer hardware depends on the ability to fabricate smaller and more precise logic devices at decreasing costs. This trend is limited by the ability to fabricate molecular logic devices and to connect them in complex patterns at the molecular level. The manufacturing technology needed will be able to economically manufacture large structures with atomic precision $/ 1$.

While the ultimate objective is economical fabrication, present capabilities preclude the manufacture of any but the most rudimentary molecular structures. Such modeling allows the rapid evaluation and retention and more intensive analysis of more promising designs. With appropriate molecular CAD software, molecular modeling software (including available computational chemistry packages, e.g., molecular mechanics, semi-empirical and $a b$ initio programs) $/ 2$, plans can be made for the development of molecular manufacturing systems on a computer.

Molecular machine proposals, specified in atomic detail can be modeled using the tools of computational chemistry. There are two modeling techniques of particular utility. The first is molecular mechanics, which utilizes empirical force fields to model the forces acting between nuclei. The second is higher order $a b$ initio calculations $/ 3 /$.

Molecular mechanics allows computational modeling of the positions and trajectories of the nuclei of individual atoms without an undue computational load. More complex analyses limit the size that the system can handle. In molecular mechanics the individual nuclei are usually treated as point masses. While quantum mechanics dictates that there must be a certain degree of positional uncertainty associated with each nucleus, this positional uncertainty is normally significantly smaller than the typical internuclear distance.

While the nuclei can reasonably be approximated as point masses, the electron cloud must be dealt with in quantum mechanical terms. Under the assumption that the position of the nuclei is the only important aspect, the quantum mechanics can be eliminated. For example, the $\mathrm{H}_{2}$ molecule involves two nuclei. While it would be possible to solve Schrodinger's equation to determine the wave function for the electrons, there is only a need to know the electronic energy as a function of the distance between the nuclei. In many systems, the impact the electrons have on nuclear position only makes a contribution to the potential energy $E$ of the system. In the case of $\mathrm{H}_{2}, E$ is a simple function of the internuclear distance $r$. The function $E(r)$ summarizes and replaces the more complex and more difficult to determine wave function for the electrons, as well as taking into account the inter-nuclear repulsion and the interactions between the electrons and the nuclei. The two hydrogen nuclei will adopt a position that minimizes $E(r)$. As $r$ becomes larger, the potential energy of the system increases and the nuclei experience a restoring force that returns them to their original distance. 
Similarly, as $\mathrm{r}$ becomes smaller and the two nuclei are pushed closer together, we also find that a restoring force pushes them farther apart, again restoring them to an equilibrium distance 14 /.

Knowing the potential energy as a function of the nuclear positions, the forces acting on the individual nuclei can be determined and therefore can compute the evolution of their position over time. The function $\mathrm{E}$ is a Newtonian potential energy function, despite the fact that the particular value of $E$ at a particular point could be computed from Schrodinger's equation. That is, the potential energy $\mathrm{E}$ is a Newtonian concept, but the particular values of $\mathrm{E}$ at particular points are determined by Schrodinger's equation.

Often called the Born-Oppenheimer approximation, this approach allows conceptual and practical simplification in the modeling of molecular systems. While it would be possible to determine $\mathrm{E}$ by solving Schrodinger's equation, in practice it is usual to use available experimental data and to infer the nature and shape of $E$ by interpolation. This approach has spawned a wide range of potential energy functions. Because the gradient of the potential energy function $\mathrm{E}$ defines a conservative force field $\mathrm{F}$, molecular mechanics methods are also called "force field" methods. While it is common to refer to "empirical force field" methods, the more recent use of ab initio methods to provide data points to aid in the design of the force fields makes this term somewhat inaccurate, though still widely used.

Of course, the "accuracy" of the force fields depends on the application. For example, such a force field would lead to serious errors in predicting the correct three-dimensional structure of a protein. Given the linear sequence of amino acids in a protein, the protein-folding problem is to determine how it will fold in three dimensions when put in solution. Often, the correct configuration will have an energy that differs from other (incorrect) configurations by a relatively modest amount, and so a force field of high accuracy is required $/ 5 /$.

While empirical force fields are accurate enough to model the behavior of chemically stable stiff structures, they do not provide sufficient accuracy to deal with chemical transitions. Thus, if we wish to model the manufacture of a molecular part then we must use higher order $a b$ initio techniques. These techniques impose severe constraints on the number of atoms that can be modeled (perhaps one or two dozen heavy atoms, depending on the hardware, software, and specific type of modeling being attempted), but can provide an accuracy sufficient to analyze the chemical reactions that must necessarily take place during the synthesis of large, atomically precise structures. Higher order $a b$ initio techniques are sufficient to analyze. the addition or removal of a small number of atoms from a specific site on a work piece. Synthesis of a large object would then consist of repeated site-specific applications of a small number of basic operations, where each basic operation changed the chemical structure of only a small number of atoms at a time. Proxided that we reject reaction mechanisms where the result predicted depends on errors that are smaller than can reasonably be modeled, the analysis of these basic operations can be satisfactorily: carried out with current methods and hardware $/ 6 \%$

While theoretical work is the first step, actually modeling a system provides invaluable information and insight about the mechanisms at work. The Materials and Process Simulation Center, California Institute of Technology in Pasadena, California, $/ 7 /$ has modeled dendrimers. Dendrimers are a new class of three- 
dimensional, man-made molecules produced by a synthetic route, which incorporates repetitive branching sequences to create a unique novel architecture. Exceptional features of the dendritic architecture include a high degree of structural symmetry, a density gradient displaying an intra-molecular minimum value and a well-defined number of terminal groups, which may be chemically different from the interior. The combination of these features creates an environment within the dendrimer molecule that facilitates an avenue to developing reliable and economical fabrication and manufacturing of functional nano-scale materials that would have properties that could be the basis of new nano-scale technology and devices.

Dendrimers and hyperbranched polymers represent a novel class of structurally controlled macromolecules derived from a branches-upon-branches structural motif. Dendrimers are well defined, highly branched macromolecules that radiate from a central core and are synthesized through a stepwise, repetitive reaction sequence that guarantees complete shells for each generation, leading to polymers that are monodisperse. The synthetic procedures developed for dendrimer preparation permit nearly complete control over the critical molecular design parameters, such as size, shape, surface/interior chemistry, flexibility, and topology. Synthetic techniques proved to be effective include the starburst divergent strategy, the convergent growth strategy and the self-assembly strategy. These methods have proved effective in generating macromolecules with a unique combination of properties. Predicting the structure, dynamics, and properties of dendrimers at the nano-scale and microscale regimes requires substantial improvements in theory (FF and simulation methodologies) and the software (the algorithms and their implementation in order to do the calculations) $/ 8 /$.

\section{IMAGING}

Laboratories are rapidly gaining atomically precise control over, and views of, matter at the atomic scale. In particular, the following microscopes/nanoscopes have been developed within the U.S.A.

Scanning probe microscopes (SPM) can image surfaces with sub-atomic precision and manipulate individual atoms and molecules on surfaces. Manipulation can be accomplished electronically, mechanically, and/or with chemically active tips. An SPM uses the interaction of a microscopic probe with the surface of a sample to measure characteristics of the sample at localized points. By scanning the sample with a probe in a two-dimensional pattern, an image can be produced. By measuring the deflection of the cantilever, often with a laser, the interaction is quantified. A feedback loop between controller and the deflection measurement system provides extreme accuracy /9/. Figure 1 shows an example if this. 


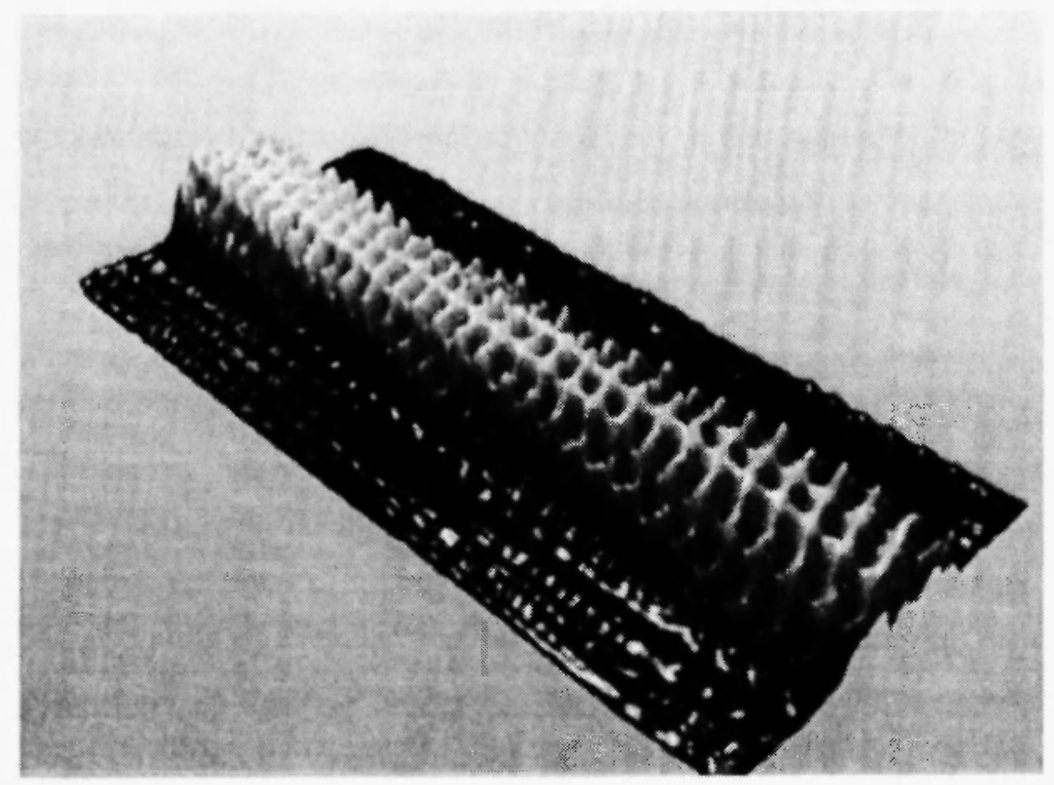

Fig. 1: Atomically precise image of a carbon nanotube /10/

Scanning tunneling microscopy (STM) involves the tunneling of electrons through vacuum from the tip of the STM to the sample. The STM can image atomic details as tiny as $1 / 25$ th the diameter of a typical atom, which corresponds to a resolution several orders of magnitude better than the best electron microscope. The STM is limited to interaction with conductors.

Atomic force microscopes (AFM) sense Van der Wall's forces from a surface, which allows measurement of nonconductive surfaces as well as operation in air and liquid. If a chemically active molecule is placed on the tip, then an AFM can be used to measure chemical forces between the tip and a surface.

"Chemical Force Microscopy" has an AFM tip coated with a hydrophilic monolayer and imaged a surface patterned with hydrophobic and hydrophilic molecules. While the surface appeared smooth to an unmodified AFM tip, the AFM was able to measure differences in frictional forces between hydrophobic and hydrophilic portions of the surface, achieving an estimated resolution of about $200 \mathrm{~nm}$. To achieve higher resolution, and measure the interaction of individual molecules with a surface, a sharper tip is necessary. The atomic precision was achieved when carbon nanotubes were attached to SPM tips. The open-ended carbon nanotubes covalently functionalized with several different molecules, to image a chemically varied surface achieving a lateral chemical resolution of approximately $3 \mathrm{~nm}$, which used $\mathrm{Si}$ and $\mathrm{Si}_{3} \mathrm{~N}_{4}(15 \mathrm{~nm})$ or multiwalled-carbon-nanotube tips. Scanning the sample with different functional groups on the tip resulted in distinctly different images and the differences could be explained on the basis of chemical affinity between the tip and the surface. Since both closed and open carbon nanotubes may be functionalized in many ways, modified nanotube probes may perform extremely well controlled chemistry at precise locations and use applied forces to overcome reaction barriers /11/. 


\section{MATERIAL INTEGRATION}

Reducing the grain size in the starting materials enhances the mechanical, electrical, and magnetic properties of ceramics, sintered metals and composites. Other properties such as strength and ductility can be enhanced either by the direct influence in an ultrafine microstructure or by the advantage gained by mixing and bonding ultrafine powders $/ 16 /$.

Several materials, such as $\mathrm{Al}_{2} \mathrm{O}_{3}$ or $\mathrm{SiC}$, have been used to increase the strength of nano-layered structures $/ 12 /$.

In one experiment, conducted at Sandia National Laboratories, alumina coatings were used. Alumina coatings with their chemical inertness have a high mechanical strength and good wear resistance to high temperatures. Alumina has poor superplastic properties and low fracture toughness. The mechanical properties are significantly enhanced by the addition of $\mathrm{SiC}$ particles. Multilayered $\mathrm{Al}_{2} \mathrm{O}_{3} / \mathrm{SiC}$ thin film samples with a good purity were deposited using a dual-source system. No significant difference was seen between the samples. However, after an annealing process, the $\mathrm{Al}_{2} \mathrm{O}_{3} / \mathrm{SiC}$ samples showed an increase in hardness when compared to the plain $\mathrm{Al}_{2} \mathrm{O}_{3}$ samples /14/.

Another experiment performed at Sandia National Laboratories looked at silicon space cells and how to better design and operate them. The main disadvantages of silicon compared to GaAs-based solar space cells are its low conversion efficiency and poor radiation tolerance. Both of these disadvantages are related to silicon's indirect-bandgap structure. Indirect-bandgap semiconductors have weak optical absorption. As a result of the weak absorption, a thick layer of silicon must be used to fully absorb light. These thick silicon cells give the satellites a large mass, which is not desired. Work has been done on integration of nano-scale textures into complete solar cells. The internal quantum efficiency, which is the number of collected electrons per absorbed photon, gives an indication of the effectiveness of the nano-scale features to enhance the absorption near the surface. The intemal quantum efficiency spectra were enhanced in the infrared spectrum due to the use of periodically textured surfaces. This process offers the possibility of enhanced radiation tolerance for silicon solar cells $/ 15 /$.

In another experiment, conducted by the Department of Materials Science and Engineering at the University of Illinois, amorphous diamonds were used for surface micromachined microelectro-mechanical systems (MEMS) and sensors. Amorphous diamonds showed improved wear resistance, strength, and fracture toughness. The experiment showed that the tensile strength and modulus of the amorphous diamond MEMS were significantly increased. These samples were tested against the current standard material for surface micromachined MEMS, polysilicon /16/.

\section{MEDICINE}

Given such molecular tools, small devices could be designed to identify and kill cancer cells. The device would have a small computer, several binding sites to determine the concentration of specific molecules. A 
supply of some poison would be selectively released with the ability to kill a cell identified as cancerous. The device would circulate freely throughout the body, and would periodically sample its environment by determining whether the binding sites were or were not occupied. Occupancy statistics would allow determination of concentration. Today's monoclonal antibodies are able to bind to only a single type of protein or other antigen, and have not proven effective against most cancers. The cancer-killing device suggested here could incorporate a dozen different binding sites and so could monitor the concentrations of a dozen different types of molecules. The computer could determine if the profile of concentrations fit a preprogrammed "cancerous" profile and would release the poison $/ 17 /$.

Beyond being able to determine the concentrations of different compounds, the cancer killer could also determine local pressure. Pressure sensors little more than 10 nanometers on a side could detect pressure changes of less than 0.1 atmospheres. Linear increases in sensor volume would produce exponential increases in immunity to thermal noise and linear improvements in pressure sensitivity. Doubling the linear dimensions of the sensor would produce an eight-fold increase in both volume and pressure sensitivity.

Using an acoustic signal in the megahertz range, the cancer killer could safely receive broadcast instructions. By using several macroscopic acoustic signal sources, the cancer killer could determine its location within the body $/ 18 \%$.

\begin{abstract}
AEROSPACE
Nanotechnology for aerospace applications is derived from the theoretical abilities of atomically precise molecular machines. While SPMs provide positional control at the atomic scale, they are too bulky to build macroscopic products atom by atom because the parallelism is limited by the size of the machines. Very large numbers of molecular machines can fit in a small space and, properly organized, could provide the parallelism necessary to build macroscopic products by positioning individual atoms. Only one such machine has been built, although several computational studies have been undertaken. A molecular rotor was observed operating on an atomically precise copper surface in ultrahigh vacuum. The molecule rotated due to thermal energy when separated from a bearing formed by a hexagonal lattice of the same molecules. Rotation stopped when the molecule moved into contact with the hexagonal lattice /19/.

Molecular nanotechnology is making great strides with respect to very small products. Placing and maintaining humans in orbit require macroscopic systems. This requires integration of vast numbers of microscopic elements. Biological systems scale up from very small beginnings by cell replication. Thus, the idea was proposed to build programmable molecular machines with the ability to self-replicate. Theory suggests that self-replicating machines could lead to fantastic productivity because of their exponential growth potential $/ 20 /$.
\end{abstract}




\section{COMPUTERS}

Computer chips are desired to be very small, 10-100 nanometers, so that microcomputers can be built to perform complex computations. Therefore, nanotechnology and manufacturing chips at nano-scale are becoming very important. Nanotechnology is based on the ability to create and utilize materials, devices and systems through control of the matter at the nanometer scale. If successful, nanotechnology is expected to lead to broad new technological developments.

The current method by which a computer chip is manufactured is lithography. Lithography is the finest manufacturing technology, but there seems to be a fundamental limit in how much further we can improve it. In lithography, fine lines are drawn on silicon wafer, which are light sensitive. Such a light sensitive film is called "resist" and is spread over the silicon wafer. The exposed resist is then washed, like photographic film, in a particular chemical, which diffuses to the silicon wafer where the resist is not washed away. Finally, the remaining resist is washed away, along with any chemicals that it prevented from reaching the silicon surface. The result is a fine pattern of some desired chemical that is laid out on the silicon surface. By repeating this process, an intricate set of interlocking patterns can be made that defines the complex logic elements of a modern computer chip. The challenge is to move beyond the scope of lithography and develop materials and manufacturing methods that allow manufacturing at molecular level.

Manufactured products are made from atoms. The properties of those products depend on how those atoms are arranged. Viewed from the molecular level today's macroscopic manufacturing methods are crude and imprecise. Casting, milling, welding, and all other traditional manufacturing methods spray atoms in great statistical heaps. Even lithography (which already lets us put millions of transistors on a chip no bigger than your fingernail) is fundamentally statistical and random. Exactly, how many dopant atoms are in a single transistor and exactly where each individual dopant atom is located is neither specified nor known; if we have roughly the right number in roughly the right place, we can make a working transistor.

On the cutting edge of computer construction is the company NanoLogic. NanoLogic, Inc. $121 /$ is a California Corporation founded in 1997 by Dr. Robert W. Schmieder. The company is currently developing a new class of computers based on nanotechnology that have the potential to be orders of magnitude more powerful than current computers. The approach taken by NanoLogic is to formulate the data into structures that match the unique electronic properties of individual nano-scale devices. Constructing arrays of such devices acting sequentially on the data can make a computer. The advantage of this new computer will be its high logic density and throughput, estimated to be two to four orders of magnitude higher than current computers. Applications include military (smart weapons, nuclear design, etc.), industrial (structural and fluid engineering, drug design, etc.), commercial (implantable personal IT aids, smart robots, etc.), and scientific (molecular dynamics, chaotic systems, etc.). 


\section{FUTURE STATEMENT OF WORK}

Arkansas Tech Univeristy will collaborate with the University of Arkansas Fayetteville and the University of Memphis to prepare samples deposit and study nano-layered structures and perform nanoindentation to determine strength properties and then study the nano-structures with AFM.

\section{CONCLUSIONS}

In summary, there is a gap in our synthetic abilities: we can make complex mechanical machinery and electronic devices including computers, which have millions of transistors, but we cannot make such devices with the precision with which a chemist can synthesize a crystal, a bio-polymer, or a relatively small molecule. With chemistry we can make precise molecular structures and compounds, but we have not been able to scale up that success to molecular computers and other macroscopic products as precise as molecules. Molecular manufacturing will, by definition, let us economically manufacture almost any specified structure that is consistent with the laws of chemistry and physics.

\section{REFERENCES}

1. R. Merkle, Computational Nanotechnology, http://www.zyvex.com/nanotech/compNano.html

2. Ibid.

3. Ibid.

4. Ibid.

5. Ibid.

6. Ibid.

7. Tahir Cagin, Guofeng Wang, Ryan Martin and William A. Goddard III, Molecular Modeling of Dendrimers for Nanoscale Applications, The Materials and Process Simulation Center, California Institute of Technology in Pasadena, California

8. Ibid.

9. A. Globus and M.R.J. Veridian, Molecular Technology in Aerospace: 1999, Technology Solutions Inc., NASA Ames Research Center in Moffett Field, California.

10. Ibid.

11. Ibid.

12. A Franks, Nanotechnology Review Article, Division of Mechanical and Optical Metrology, National; Physics Lab, Teddington, Middlesex TW110LW, UK 
13. R. Lappalainen, Nanolayered $\mathrm{Al}_{2} \mathrm{O}_{3} / \mathrm{Sl}$ C Structures Tested by Microtensile Testing, Dept. of Physics, University of Helsinki, Finland

14. Ibid

15. J.M. Gee, S.H. Zaidi and D.S. Ruby, Advanced Silicon Space Solar Cells Using Nanotechnology, Sandia National Laboratories, Albuquerque, New Mexico and Center for High Technology Materials, University of New Mexico, Albuquerque, New Mexico

16. D.A. LaVan, R.J. Hohlfelder, J.P. Sullivan, T.A. Friedmann and M. Mitchell, Tensile Properties of Amorphous Diamond Films, Sandia National Laboratories, Albuquerque, New Mexico

17. R. Merkle, in: Nanotechnology and Medicine, http://www.zvvex.com/nanotech/ nanotechAndMedicine.html

18. Ibid.

19. A. Globus and M.R.J. Veridian, Molecular Technology in Aerospace: 1999, Technology Solutions Inc., NASA Ames Research Center in Moffett Field, California.

20. Ibid

21. NanoLogic, Inc., TriValley Technology Enterprise Center Lawrence Livermore Laboratory, Livermore, CA 94550, http://www.nanologicinc.com/index.html 